Draft VERsion OCtOBER 7, 2018

Preprint typeset using $\mathrm{LATEX}_{\mathrm{E}}$ style emulateapj v. 10/09/06

\title{
THE PROTO-NEUTRON STAR PHASE OF THE COLLAPSAR MODEL AND THE ROUTE TO LONG-SOFT GAMMA-RAY BURSTS AND HYPERNOVAE
}

\author{
L. Dessart ${ }^{1}$, A. Burrows ${ }^{1}$, E. Livne ${ }^{2}$, and C.D. Otт ${ }^{1}$ \\ Draft version October 7, 2018
}

\begin{abstract}
Recent stellar evolutionary calculations of low-metallicity massive fast-rotating main-sequence stars yield iron cores at collapse endowed with high angular momentum. It is thought that high angular momentum and black hole formation are critical ingredients of the collapsar model of long-soft $\gamma$-ray bursts (GRBs). Here, we present 2D multi-group, flux-limited-diffusion MHD simulations of the collapse, bounce, and immediate post-bounce phases of a $35-\mathrm{M}_{\odot}$ collapsar-candidate model of Woosley \& Heger. We find that, provided the magneto-rotational instability (MRI) operates in the differentially-rotating surface layers of the millisecond-period neutron star, a magnetically-driven explosion ensues during the proto-neutron star phase, in the form of a baryon-loaded non-relativistic jet, and that a black hole, central to the collapsar model, does not form. Paradoxically, and although much uncertainty surrounds stellar mass loss, angular momentum transport, magnetic fields, and the MRI, current models of chemically homogeneous evolution at low metallicity yield massive stars with iron cores that may have too much angular momentum to avoid a magnetically-driven, hypernova-like, explosion in the immediate post-bounce phase. We surmise that fast rotation in the iron core may inhibit, rather than enable, collapsar formation, which requires a large angular momentum not in the core but above it. Variations in the angular momentum distribution of massive stars at core collapse might explain both the diversity of Type Ic supernovae/hypernovae and their possible association with a GRB. A corollary might be that, rather than the progenitor mass, the angular momentum distribution, through its effect on magnetic field amplification, distinguishes these outcomes.

Subject headings: MHD - stars: neutron - stars: supernovae: general - neutrinos - rotation - Gamma-
\end{abstract} ray: bursts

\section{INTRODUCTION}

There is mounting observational evidence for the association between long-soft $\gamma$-ray Bursts (GRBs) and broad-lined Type Ic supernovae (SNe; see Woosley \& Bloom 2006 for a review). Such hydrogen-deficient (and, perhaps, also helium-deficient) progenitors are compact and, if fast rotating in their core at collapse, fulfill critical requirements for the formation of a collapsar (Woosley 1993). The engine that converts energy from long-term accretion of disk material onto the black-hole (BH) may power a relativistic jet in the excavated polar regions. The jet breaks out of the progenitor surface while equatorial accretion continues. Depending on the BH mass and the angular momentum budget in the collapsing envelope, this "engine" may operate for seconds, i.e. as long as typical long-soft GRBs. Accompanying this beamed relativistic polar jet might be a disk wind, fueled by neutrinos or MHD processes, which would explode the WolfRayet envelope. This explosion and the radioactive ${ }^{56} \mathrm{Ni}$ material produced might lead to very energetic, broadlined, Type Ic SN of the hypernova variety (Iwamoto et al. 1998; MacFadyen \& Woosley 1999; Hjorth et al. 2003; Stanek et al. 2003).

State-of-the-art radiation-hydrodynamics simulations including a sophisticated equation of state (EOS) and detailed neutrino transport (Buras et al. 2003; Bur-

\footnotetext{
${ }^{1}$ Department of Astronomy and Steward Observatory, The University of Arizona, Tucson, AZ 85721; luc@as.arizona.edu,burrows@as.arizona.edu,cott@as.arizona.edu

2 Racah Institute of Physics, The Hebrew University, Jerusalem, Israel; eli@frodo.fiz.huji.ac.il
}

rows et al. 2006,2007a; Kitaura et al. 2006; Marek \& Janka 2007; Mezzacappa et al. 2007) suggest that while the neutrino mechanism of supernova explosions may work for the lower-mass massive progenitors, it may not for the more massive progenitors, characterized by an ever higher post-bounce accretion rate onto the protoneutron star (PNS). Burrows et al. (2006,2007a) have suggested that an acoustic mechanism will work for all slowly rotating progenitors that do not explode by other means within the first second after bounce. However, massive star cores endowed with a large angular momentum at the time of collapse should experience the magneto-rotational instability (MRI; Balbus \& Hawley 1991; Akiyama et al. 2003; Pessah et al. 2006; Shibata et al. 2006; Etienne et al. 2006), with the potential to exponentially amplify weak initial fields on a rotation timescale. The saturation values of such fields are ultimately set by the free-energy of differential rotation available in the surface layers of the PNS (Ott et al. 2006 ), and can be large, i.e., on the order of $10^{15} \mathrm{G}$ at a radius of a few tens of kilometers. The corresponding magnetic stresses at the neutron star surface lead systematically to powerful jet-like explosions $\sim 100 \mathrm{~ms}$ after bounce (see, e.g., Ardeljan et al. 2005; Yamada \& Sawai 2004; Kotake et al. 2004; Sawai et al. 2005; Moiseenko et al. 2006; Obergaulinger et al. 2006; Burrows et al. 2007b, hereafter B07; Dessart et al. 2007).

In this letter, we investigate, in the context of the collapsar model, the potential implications of this magnetic explosion mechanism. Our study focuses on the immediate post-bounce phase, whose importance was emphasized by Wheeler et al. $(2000,2002)$. This is in contrast to 
TABLE 1

Properties of our TWO MHD-VULCAN/2D Simulations of THE 35OC COLLAPSAR MODEL OF WH06.

\begin{tabular}{|c|c|c|c|c|c|c|c|}
\hline & $\begin{array}{c}t_{\text {end }} \\
\text { ms }\end{array}$ & $\begin{array}{c}t_{0} \\
\mathrm{~ms}\end{array}$ & $\begin{array}{l}\mathrm{M}_{10} \\
\mathrm{M}_{\odot}\end{array}$ & $\begin{array}{c}\mathrm{P}_{10} \\
\mathrm{~ms}\end{array}$ & $\begin{array}{c}\mathrm{E}_{\text {expl }} \\
\mathrm{B}\end{array}$ & $\underset{\mathrm{B} \mathrm{s}^{-1}}{\dot{\mathrm{E}}_{\text {gas }}} \dot{\mathrm{E}}_{\overrightarrow{\mathrm{E}} \times \overrightarrow{\mathrm{B}}}$ & $\begin{array}{c}v_{\max } \\
\mathrm{km} \mathrm{s}^{-1}\end{array}$ \\
\hline M0 & 369 & $\ldots$ & 2.1 & 4 & 0.03 & 0.25 & 43,000 \\
\hline M1 & 666 & 349 & 1.7 & 12 & 3.31 & 3.0 & 58,000 \\
\hline
\end{tabular}

Note. $-t_{\text {end }}$ gives the time at the end of each simulation, while $t_{0}$ is the time when the rate of polar mass ejection first overcomes equatorial mass accretion. All quoted quantities in the table correspond to the final time in each simulation, while times are given with respect to core bounce. $\mathrm{M}_{10}\left(\mathrm{P}_{10}\right)$ corresponds to the total baryonic mass (average rotation period) inside the $10^{10} \mathrm{~g} \mathrm{~cm}^{-3}$ isodensity contour. $\dot{\mathrm{E}}_{\text {gas }}\left(\dot{\mathrm{E}}_{\overrightarrow{\mathrm{E}} \times \overrightarrow{\mathrm{B}}}\right)$ is the Bernoulli (Poynting) power in the ejecta, obtained by integrating the corresponding flux over a shell with a radius of $500 \mathrm{~km}$. [See text for additional information.]

previous work which explored only the phase subsequent to BH formation (MacFadyen \& Woosley 1999; Aloy et al. 2001; Zhang et al. 2003; Proga 2005). Indeed, two terms sometimes used in the collapsar context are "failed SN" (MacFadyen \& Woosley 1999) and "prompt BH formation" (MacFadyen et al. 2001). Our analysis supports the idea that the conditions for the collapsar model, as stated so far, are also suitable for a magnetically-driven explosion in the immediate post-core-bounce PNS phase, and that $\mathrm{BH}$ formation may be so delayed for a range of putative progenitor models that it does not in fact occur $^{3}$. In $\S 2$, we present radiation MHD simulations with the code VULCAN/2D (Livne et al. 2004,2007) of a collapsar-candidate model that support this thesis. In $\S 3$, we discuss the implications of our results for stellar evolutionary models that might lead to collapsars and/or hypernovae.

\section{MODEL AND RESULTS}

We present results from two-dimensional, rotating, multi-group, flux-limited diffusion magnetohydrodynamics simulations, using VULCAN/2D (Livne et al. 2004,2007; see also appendices in Dessart et al. 2006 and Burrows et al. 2007a), of the 35OC progenitor model and collapsar candidate of Woosley \& Heger (2006; hereafter WH06). The numerical procedure we follow is identical to that of B07 in every respect, except the choice of progenitor. WH06's model is evolved from a $35 \mathrm{M}_{\odot}$ zero-age main sequence star endowed with a total angular momentum of $1.4 \times 10^{53} \mathrm{erg} \cdot \mathrm{s}$, a metallicity of $1 \%$ the solar value, and a reduction by a factor of 10 in the prescribed mass loss rates during the Wolf-Rayet phase. Our simulations extend out to a maximum radius of $5000 \mathrm{~km}$ (which contains $\sim 3 \mathrm{M}_{\odot}$ ) and cover a $90^{\circ}$ quadrant, bounded by the rotation axis and the equator. We adopt WH06's initial rotational, density, temperature, and electron-fraction profiles for that progenitor. For the magnetic field distribution, we start with magnitudes and morphology that are consistent with the 35OC model of WH06. We use a field uniform

\footnotetext{
3 In the present context, $\mathrm{BH}$ formation is never prompt, since it takes a finite time, on the order of seconds, for the PNS to accumulate the critical mass at which it experiences the gravitational instability. This is in contrast with super-massive stars, such as the progenitors of pair-instability SNe, which may form an apparent horizon during collapse and thus "directly" transition to a BH (Liu et al. 2007).
}

within $3000 \mathrm{~km}$, and dipolar beyond. In our reference model, M0, we employ initial poloidal and toroidal field magnitudes of $2 \times 10^{10} \mathrm{G}$ and $8 \times 10^{11} \mathrm{G}$, respectively, in close quantitative agreement with the $35 \mathrm{OC}$ model of WH06. However, we also study a model, M1, with an initial poloidal field that is five times stronger. This leads to a magnetic field energy at $\sim 100 \mathrm{~ms}$ after core bounce that is closer to the value expected at the PNS surface, were we to adequately resolve the MRI (B07). In Table 1, we give important quantities characterizing the two simulations performed. Note that if magnetic fields are ignored in the pre-collapse evolutionary calculations of WH06, core angular velocities reach $5-22 \mathrm{rads}^{-1}$, much larger than the $1.98 \mathrm{rad} \mathrm{s}^{-1}$ achieved in the $35 \mathrm{OC}$ model. A bounce at sub-nuclear densities may ensue and lead to BH formation (Akiyama \& Wheeler 2005). Ignoring magnetic torques in the models most prone to magnetic-field generation during the pre-collapse phase seems inconsistent. Thus, we focus on the more slowly rotating progenitors, evolved with magnetic fields, which inevitably bounce at nuclear densities.

After an initial collapse phase that lasts $\sim 245 \mathrm{~ms}$, the central density reaches $\sim 3 \times 10^{14} \mathrm{~g} \mathrm{~cm}^{-3}$, the EOS stiffens, a shock is born, and propagates outward, but is debilitated by the photo-dissociation of the infalling outer iron-core and the burst of electron neutrinos. The shock stalls at $\sim 150 \mathrm{~km}$, and within a few tens of ms after bounce, it becomes increasingly aspherical. The net gain from neutrino emission and absorption processes, the entropy, and the material accretion rates get progressively larger at larger latitudes as the degree of oblateness of the fast-rotating PNS increases. Subsequent to the amplification due to compression by a factor of $\sim 2500$ in both magnetic field components, the toroidal magnetic field increases after bounce due to the winding of the poloidal field component. At the same time, accretion of the outer magnetized core continues and enhances the total magnetic field energy. By $150 \mathrm{~ms}$ (300 ms) after bounce, the magnetic pressure at the surface of the PNS along the pole in the M1 (M0) model is comparable to the gas pressure, and a bipolar, magnetically-driven, baryon-loaded, and non-relativistic jet is initiated, reversing accretion into ejection along the polar direction. As shown in the left panel of Fig. 1 the initial jet mass-loss rate is only $\sim 0.01 \mathrm{M}_{\odot} \mathrm{s}^{-1}$, but in the M1 model and by $\sim 350 \mathrm{~ms}$ after bounce it exceeds the accretion rate. At this time, the accumulated baryonic PNS mass is only $1.93 \mathrm{M}_{\odot}$ (middle panel of Fig. 1), and, thus, well below the $2.17 \mathrm{M}_{\odot}$ baryonic mass transition to $\mathrm{BH}$ formation that we derive with our Shen EOS (Shen et al. 1998). Note that for a solidbody rotator this limit may increase by up to $10-20 \%$ (Cook et al. 1994), but for a differentially-rotating neutron star this limit may be considerably larger (Baumgarte et al. 2000). Hence, rotation, and in particular differential rotation, enhances the potential for explosion during a PNS phase. By $650 \mathrm{~ms}$ after bounce, the explosion energy in the M1 model reaches $\sim 3.3 \mathrm{~B}\left(10^{51} \mathrm{erg} \equiv\right.$ 1 Bethe [1 B]) (see right panel of Fig. 1), although due to the continued accretion along near-equatorial latitudes a quasi-steady-state is reached with an explosion power sustained at $\sim 10 \mathrm{~B} \mathrm{~s}^{-1}$. As shown in Fig. 2, the jet resembles a magnetic tower (Lynden-Bell 2003; Uzdenski \& MacFadyen 2001), but is confined primarily by the ram pressure of the infalling dense envelope (B07). As 

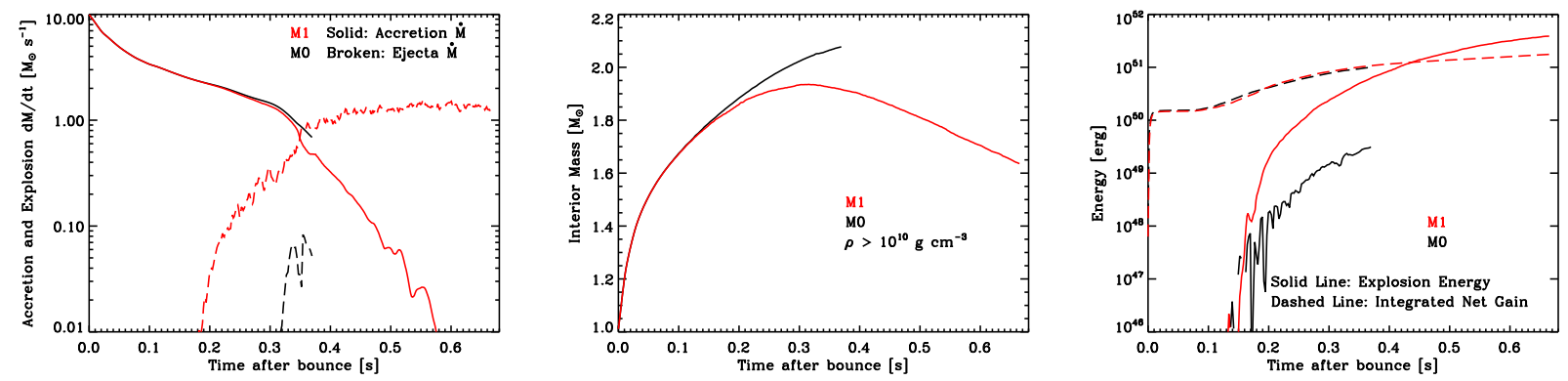

FIG. 1. - Left: Time evolution of the instantaneous integrated mass flux accreting (solid line) and outflowing (dashed lines) through a shell at a radius of $500 \mathrm{~km}$, for models M0 (black) and M1 (red). Middle: Same as left, but for the total mass interior to the iso-density contour corresponding to $10^{10} \mathrm{~g} \mathrm{~cm}^{-3}$. Right: Same as left, but for the explosion energy (solid line) and the net integrated neutrino gain (dashed lines) outside the high-density regions bounded by the $10^{10} \mathrm{~g} \mathrm{~cm}^{-3}$ contour. [See text for discussion.]

time progresses, its base broadens and, given the quasisteady jet conditions, the mass ejection rate grows and the accretion is limited to progressively smaller latitudes. Both cause and effect, the increase in ejecta volume enhances the neutrino contribution to the explosion energy, although it remains a subdominant part of the total by the end of the simulation. Extraction of core rotational energy by magnetic torques is also in evidence in the M1 model from the increase in the average PNS rotation period $^{4}$ from 8 to $12 \mathrm{~ms}$ between $200 \mathrm{~ms}$ and $600 \mathrm{~ms}$ after bounce, while over half this interval the average period decreases from 5 to $4 \mathrm{~ms}$ in the weakly exploding M0 model. The decrease in the free energy of rotation in the M1 model is on the order of $3 \mathrm{~B}$, and is comparable to the magnitude of the explosion energy. This supports the idea that core rotation energy fuels the magneticallydriven ejecta. Hence, the M1 model, modified slightly to yield fields at saturation that agree roughly with what would obtain in the presence of the MRI, boasts a clear and powerful explosion. In this model, and once the explosion is well established, the PNS loses mass at a steady rate, and has a mass of only $\sim 1.7 \mathrm{M}_{\odot}$ at the end of the simulation. The broadening of the base of the jet suggests that the explosion will not choke (nor induce any significant fallback), and encompassing a larger solid angle, will instead lead to explosion in all directions. Hence, such an object is unlikely ever to transition to a $\mathrm{BH}$ and to lead to a collapsar.

By contrast, in the M0 model, the explosion emerges later, when the neutron star baryonic mass has already accumulated $\sim 2.1 \mathrm{M}_{\odot}$, and thence may be susceptible to collapse to a $\mathrm{BH}$. The free-energy of core rotation has been partially tapped, but the potential subsequent powering of a GRB may not be compromised. This model, by mimicking more slowly rotating cores or an inefficient MRI, offers a limiting case for the formation or non-formation of a $\mathrm{BH}$, and a possible collapsar.

\section{DISCUSSION}

The potential for exponential growth on a rotational timescale of initial seed magnetic fields by the MRI (Shibata et al. 2006; Etienne et al. 2006), fueled by the free energy of core rotation, makes the initial angular momentum budget of the progenitor star the key parameter in determining the outcome during the immediate post-

4 We define the average rotation-period as the period of the rigidly-rotating PNS that has the same total angular momentum and structure inside the $10^{10} \mathrm{~g} \mathrm{~cm}^{-3}$ isodensity contour. bounce phase (B07). A magnetically-driven, baryonloaded, and non-relativistic, explosion is obtained for WH06's 35OC collapsar candidate model, evolved at low metallicity from a $35 \mathrm{M}_{\odot}$ fast-rotating main-sequence star. The explosion occurs $\sim 200 \mathrm{~ms}$ after bounce and reaches $\sim 3 \mathrm{~B} \sim 400 \mathrm{~ms}$ later. After an initial accretion phase, the steadily decreasing PNS mass reaches only $\sim 1.7 \mathrm{M}_{\odot}$ at the end of the simulation, and, thus, the quasi-steady explosion we observe suggests that $\mathrm{BH}$ formation is unlikely to occur. Moreover, baryon contamination prevents the ejecta from becoming relativistic. Note that the production of a GRB in the collapsar context is contingent on the gravitational collapse of the PNS to a BH.

The recent stellar evolutionary calculations of Yoon \& Langer (2005), WH06, and Meynet \& Maeder (2007) of fast-rotating main-sequence objects at low metallicity systematically predict such fast-rotating cores at collapse. Starting from similar conditions for a $35-40 \mathrm{M}_{\odot}$ star, but using different mass-loss "recipes," they obtain very similar rotational profiles in the inner core. Allowing for anisotropic mass loss (Meynet \& Maeder 2007), a model of C. Georgy (2007, priv. comm.) suggests an even larger (by a factor of two) specific angular momentum in the inner $3 \mathrm{M}_{\odot}$ at the end of silicon core burning. Despite the agreement between these different evolutionary computations, the magnetically-driven explosion and the "failed" $\mathrm{BH}$ formation described here are conditional on the uncertain treatment of mass loss, angular momentum transport, and magnetic processes (Spruit 2002) during the pre-collapse evolution.

At very low metallicities, radiatively-driven winds of massive stars are inhibited by the lack of metals $(\mathrm{Ku}-$ dritzki 2002; Vink et al. 2001; Vink \& de Koter 2005), whose optically-thick lines intercept radiation momentum (Castor et al. 1975). Recent revisions downward of mass-loss rates due to clumping (Owocki et al. 1988; Bouret et al. 2005; Fullerton et al. 2006) suggest, however, the potential importance of episodic outbursts, akin to the 1843 giant eruption of Eta Carina (Smith \& Owocki 2006). The metallicity dependence of such phenomena is entirely unknown, mostly because the fundamental cause of the outburst remains a mystery. While line driving seems excluded, continuum driving of a porous medium at super-Eddington luminosities has been proposed by Owocki et al. (2004) as an alternative. Finally, mass loss in fast-rotating, and sometimes critically-rotating (Townsend et al. 2004), envelopes 


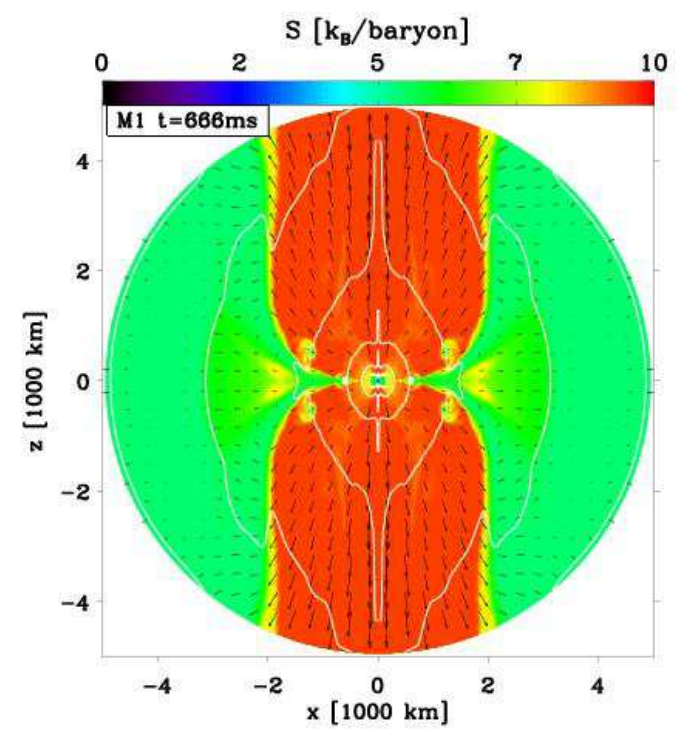

FIG. 2.- Colormap of the entropy at $666 \mathrm{~ms}$ after bounce for model M1, overplotted with white iso-density contours (every decade downward from $10^{10} \mathrm{~g} \mathrm{~cm}^{-3}$ ) and velocity vectors (length saturated to $15 \%$ of the width of the display and corresponding to a velocity of $30,000 \mathrm{~km} \mathrm{~s}^{-1}$.)

is complicated by the effects associated with centrifugal support, surface oblateness, and gravity-darkening (Cranmer \& Owocki 1995; Owocki et al. 1996), so that the mass-loss "recipes" used in stellar evolutionary models are not always substantiated by observational and theoretical evidence. At present, and in light of our simulations, it appears that chemically-homogeneous evolution of fast-rotating main-sequence massive stars at low metallicity systematically yields iron cores at collapse that may have too much angular momentum, a property that prevents the formation of a collapsar. Uncertainties in the modeling of the pre-collapse evolution may result, however, in slower-rotating iron $\operatorname{cores}^{5}$ and, thus, might inhibit an early magnetically-driven explosion in favor of black hole, and perhaps collapsar, formation.

We conclude that variations in the angular momentum distribution of pre-collapse massive stars may lead to different post-bounce scenarios. Non- or slowly-rotating progenitors may explode with weak/moderate energy $(\lesssim 1 \mathrm{~B})$ through a neutrino or an acoustic mechanism $\lesssim 1 \mathrm{~s}$ after bounce, or may collapse to a $\mathrm{BH}$. Objects with large angular momentum in the envelope, but little in the core, may proceed through the PNS phase, transition to a $\mathrm{BH}$ and form a collapsar with a GRB signature. Owing to the modest magnetic-field amplification above the PNS, a weak precursor polar jet may be launched, soon overtaken by a baryon-free, collimated relativistic jet. At the same time, the progenitor envelope is exploded by a disk

\footnotetext{
5 Note that the rotational energy $E_{\text {rot }}$ is a stiff function of angular velocity $w$, i.e., $E_{\text {rot }} \propto w^{2}$.
}

wind, resulting in a hypernova-like $\mathrm{SN}$ with a large luminosity (large ${ }^{56} \mathrm{Ni}$ mass). Finally, and this is what we conclude here, objects with large angular momentum in the core may not transition to a BH. Instead, and fueled by core-rotation energy, a magnetically-driven baryonloaded non-relativistic jet is obtained without any GRB signature. The explosion has the potential of reaching energies of a few B to $10 \mathrm{~B}$, and for viewers along the poles of looking like a Type Ic hypernova-like SN with broad lines. For a viewer at lower latitudes, the delayed and less energetic explosion nearer the equator may look more like a standard Type Ic SN (Höflich et al. 1999). This volume-restricted jet-like explosion is dimmer, as the amount of processed ${ }^{56} \mathrm{Ni}$ may be significantly less than the $\sim 0.5 \mathrm{M}_{\odot}$ obtained in the collapsar context (MacFadyen \& Woosley 1999). Hence, magnetic processes during the post-bounce phase of fast-rotating iron cores offer a potential alternative to collapsar formation and long-soft GRBs by producing non-relativistic non-Poynting-flux-dominated baryon-loaded hypernovalike explosions without any GRB signature. Importantly, while our study narrows the range over which the collapsar model may exist, it also offers additional routes to explain the existence of GRB/SN-hypernova events like SN 1998bw (Woosley et al. 1999), and hypernova events like SN 2002ap without a GRB signature (Mazzali et al. 2002).

More generally, magnetic effects should naturally arise in the context of gravitational collapse and fast rotation. The resulting angular momentum of newly-formed $\mathrm{BHs}$ and magnetars, for example, would be reduced, perhaps considerably, by any prior magnetically-driven explosion, and, thus, may decrease the power of subsequent mass ejections from compact objects (see, e.g., Thompson et al 2004).

We thank Cyril Georgy, Norbert Langer, and SungChul Yoon for providing their stellar evolutionary models, for a comparison with the 35OC model of WH06 used in this work. We also thank Stan Woosley for fruitful discussions and insight. We acknowledge support for this work from the Scientific Discovery through Advanced Computing (SciDAC) program of the DOE, under grant numbers DE-FC02-01ER41184 and DE-FC0206ER41452, and from the NSF under grant number AST0504947. E.L. thanks the Israel Science Foundation for support under grant \# 805/04, and C.D.O. thanks the Joint Institute for Nuclear Astrophysics (JINA) for support under NSF grant PHY0216783. This research used resources of the National Energy Research Scientific Computing Center, which is supported by the Office of Science of the U.S. Department of Energy under Contract No. DE-AC03-76SF00098.

\section{REFERENCES}

Akiyama, S., et al. 2003, ApJ, 584, 954

Akiyama, S. \& Wheeler, J.C. 2005, ApJ, 629, 414

Ardeljan, N.V., et al. 2005, MNRAS, 359, 333

Balbus, S. A., \& Hawley, J. F. 1991, ApJ, 376, 214

Baumgarte, T. W., et al. 2000, ApJ, 528, L29
Bouret, J. C., Lanz, T., \& Hillier, D. J. 2005, A\&A, 438, 301

Burrows, A., et al. 2006, ApJ, 640, 878

Burrows, A., et al. 2007a, ApJ, 655, 416

Burrows, A., et al. 2007b, ApJ, 664, 416 (B07)

Castor, J. I., Abbott, D. C., \& Klein, R. I. 1975, ApJ, 195, 157 
Cook, G. B., et al. 1994, ApJ, 424, 823

Cranmer, S. R., \& Owocki, S. P. 1995, ApJ, 440, 308

Dessart, L., Burrows, A., Livne, E., \& Ott, C. 2006, ApJ, 645, 534

Dessart, L., Burrows, A., Livne, E., \& Ott, C. 2007, ApJ, 669, 585

Etienne, Z. B., et al. 2006, Phys. Rev. D, 74, 044030

Fullerton, A. W., et al. 2006, ApJ, 637, 1025

Hjorth, J., et al. 2003, Nature, 423, 847

Höflich, P., Wheeler, J. C., \& Wang, L. 1999, ApJ, 521, 179

Iwamoto, K., et al. 1998, Nature, 395, 672

Kitaura, F. S., et al. 2006, A\&A, 450, 345

Kotake, K., Sawai, H., Yamada, S., \& Sato, K. 2004, ApJ, 608, 391

Kudritzki, R. P. 2002, ApJ, 577, 389

Langer, N., et al. 1999, ApJ, 520, L49

Liu, Y. T., Shapiro, S. L., \& Stephens, B. C. 2007, Phys. Rev. D, 76,084017

Livne, E. et al. 2004, ApJ, 609, 277

Livne, E., et al. 2007, ApJS, 170, 187

Lynden-Bell, D. 2003, MNRAS, 341, 1360

MacFadyen, A. I., \& Woosley, S. E. 1999, ApJ, 524, 262

MacFadyen, A. I., Woosley, S. E., \& Heger, A. 2001, ApJ, 550, 410

Marek, A., \& Janka, H.-T. 2007, arXiv:0708.3372

Mazzali, P. A., et al. 2002, ApJ, 572, L61

Meynet, G., \& Maeder, A. 2007, A\&A, 464, L11

Mezzacappa, A., et al. 2007, American Institute of Physics Conference Series, 924, 234

Moiseenko, S.G., et al. 2006, MNRAS, 370, 501

Obergaulinger, M., Aloy, M. A., Müller, E. 2006, A\&A, 450, 1107

Ott, C. D., et al. 2006, ApJS, 164, 130

Owocki, S.P., Castor, J.I., \& Rybicki, G.B. 1988, ApJ, 335, 914

Owocki, S.P., Cranmer, S.R., \& Gayley, K.G. 1996, ApJ, 472, L115
Owocki, S. P., Gayley, K.G., \& Shaviv, N. J. 2004, ApJ, 616, 525 Paczynski, B. 1990, ApJ, 363, 218

Pessah, M. E., Chan, C.-K., \& Psaltis, D. 2006, MNRAS, 372, 183 Proga, D. 2005, ApJ, 629, 397

Sawai, H., Kotake, K., \& Yamada, S. 2005, ApJ, 631, 446

Shen, H., Toki, H., Oyamatsu, K., \& Sumiyoshi, K. 1998, Neutron Stars and Pulsars: Thirty Years after the Discovery, 157

Shibata, M., et al. 2006, Phys. Rev. D, 74, 104026

Smith, N., \& Owocki, S. P. 2006, ApJ, 645, L45

Spruit, H. C. 2002, A\&A, 381, 923

Stanek, K. Z., et al. 2003, ApJ, 591, L17

Thompson, T. A., Chang, P., \& Quataert, E. 2004, ApJ, 611, 380

Townsend, R.H.D., et al. 2004, MNRAS, 350, 189

Uzdensky, D. A., \& MacFadyen, A. I. 2006, ApJ, 647, 1192

Vink, J. S., et al. 2001, A\&A, 369, 574

Vink, J. S., \& de Koter, A. 2005, A\&A, 442, 587

Wheeler, J. C., Yi, I., Höflich, P., \& Wang, L. 2000, ApJ, 537, 810

Wheeler, J. C., Meier, D. L., \& Wilson, J. R. 2002, ApJ, 568, 807

Woosley, S. E. 1993, ApJ, 405, 273

Woosley, S. E., et al. 1999, ApJ, 516, 788

Woosley, S. E., \& Heger, A. 2006, ApJ, 637, 914 (WH06)

Woosley, S. E., \& Bloom, J. S. 2006, ARA\&A, 44, 507

Yamada, S., \& Sawai, H. 2004, ApJ, 608, 907

Yoon, S.-C., Langer, N., \& Norman, C. 2006, A\&A, 460, 199

Yoon, S.-C., \& Langer, N. 2005, A\&A, 443, 643

Zhang, W., Woosley, S. E., \& MacFadyen, A. I. 2003, ApJ, 586, 356 